spatial retro-cues were found to increasingly benefit the observers when the number of items in the array increased.

Recordings of event-related potentials (ERPs) also revealed brain potentials related to search in VSTM. Nobre et al. (2008) found a brain potential and labeled it the $\mathrm{N}_{\mathrm{RS}}$, which showed systematic changes in amplitude and duration that mirrored the behavioral findings during search. Interestingly, the ERP covaried with the degree of search required during the neutral retro-cue trials in which subjects did not benefit from retrodictive spatial cues.

Overall, the findings add support to the idea that voluntary attention can influence internal mental processing, like search through VSTM. Models that argue for immutable storage of VSTM are not compatible with the findings. However, the research suggests that William James' intro- spections on attention are as rich as they appear at first reading, and that the focus of the mind's eye can be turned inward, to affect internal mental processes, as well as outward to modulate the processing of sensory inputs from the world around us.

\section{REFERENCES}

Cherry, E. C. (1953). Some experiments on the recognition of speech, with one and two ears. J. Acoust. Soc. Am. 25, 975-979.

Desmedt, J., and Robertson, D. (1977). Differential enhancement of early and late components of the cerebral somatosensory evoked potentials during forced-paced cognitive tasks in man. J. Physiol. 271, 761-782.

Hillyard, S., Hink, R., Schwent, V., and Picton, T. (1973). Electrical signs of selective attention in the human brain. Science 182, 177-180.

James, W. (1890). Principles of Psychology. New York, H. Holt.

Mangun, G. R., and Hillyard, S. A. (1991). Modulation of sensory-evoked brain potentials provide evidence for changes in perceptual processing during visual-spatial priming. J. Exp. Psychol. Hum. Percept. Perform. 17, 1057-1074.

Nobre, A. C., Griffin, I. C., and Rao, A. (2008). Spatial attention can bias search in visual short-term memory. Front. Hum. Neurosci. 1, 1-9.

Posner, M. I. (1978). Chronometric Explorations of Mind. Oxford, UK, Lawrence Erlbaum.

Van Voorhis, S., and Hillyard, S. (1977). Visual evoked potentials and selective attention to points in space. Percept. Psychophys. 22, 54-62.

Zhang, W., and Luck, S. (2008). Discrete fixed representations in visual working memory. Nature 453, 233-235.

Received: 22 October 2008; published: 15 December 2008

Citation: Front. Neurosci. (2008) 2, 2: 133-134. doi: 10.3389/neuro.01.044.2008

Copyright: () 2008 Mangun. This is an open-access publication subject to an exclusive license agreement between the authors and the Frontiers Research Foundation, which permits unrestricted use, distribution, and reproduction in any medium, provided the original authors and source are credited.

\title{
Adding complexity to emotion-cognition interactions: the stressed individual
}

\section{Carmen Sandi*}

Brain Mind Institute, Ecole Polytechnique Federale de Lausanne, Lausanne, Switzerland

*Correspondence: sandi@epfl.ch

\section{A commentary on}

Emotion and cognition in high and low stress-sensitive mouse strains: a combined neuroendocrine a behavioral study in $\mathrm{BALB} / \mathrm{c}$ and $\mathrm{C} 57 \mathrm{BL} / 6 \mathrm{~J}$ mice

by Vera Brinks, Maaike van der Mark, Ronald de Kloet and Melly Oitzl

Emotion and cognition were not long ago considered as independent brain functions with respective underlying neural systems working in parallel and only occasionally interacting. This earlier perspective considered that the main focus of Behavioral Neuroscience was to understand the neurobiological basis of cognition, which was considered as a "cold" mode of brain functioning. "Hot" emotional systems were regarded as inferior and not central for the eventual understanding of how the human brain works. This view has drastically changed in the past two decades with an increasing number of studies pointing out not only to a close interaction between emotion and cognition, but also to their true integration in the production of behavior (Pessoa, 2008).

Fearful experiences usually become part of our strongest memories. The uneasiness we feel when having difficulties to take a decision in a family-relevant conflict can greatly determine our ability to eventually choose an appropriate solution. These are just two examples of how the intricacy between the so-called emotional and cognitive functions really operates together. In recent years, much progress has been done both on the phenomenological characterization of such interplay and on the identification of the neural circuits and molecules that sustain cognitive-emotional behaviors (Phelps and LeDoux, 2005; Richter-Levin and Akirav, 2003); however, there are at least two key issues to understand the whole picture that has been so far largely disregarded. One issue is the key standing role of stress systems. The other issue is the question of individual dif- ferences and how the differences can affect stress-cognitive-emotional interactions.

In a research report published in Frontiers in Behavioral Neuroscience, Brinks et al. (2007) have specifically addressed these two key issues. The authors took advantage of the known drastic differences in the responsiveness of stress systems in two inbred strains of mice, $\mathrm{BALB} / \mathrm{c}$ and $\mathrm{C} 57 \mathrm{BL} / 6 \mathrm{~J}$ - respectively high and low stress-responsive - and studied how the differences were related to neuroendocrine and behavioral responses of the mice to emotion- and cognition-biased situations. The authors also studied how exposure to predator stress affected both behavioral and neuroendocrine responses. The study revealed intriguing relationships between the expression of the two corticosteroid receptors in brain regions typically involved in the emotion-cognition interplay (hippocampus, amygdala, and prefrontal cortex), animals' glucocorticoid reactivity, and the performance and learning strategy followed when learning a spatial task. 
A clear asset of the study was the application of multivariate statistics - Principal Component Analysis - to assess, in an integrated manner, the interrelationships between variables related to stress, emotion, and cognition, with the aim of underscoring the sets of variables that form coherent subsets. The advantage of the approach over simpler correlational analyses is clear when exploring the nature of interactions in functional systems, which as stress, emotion, and cognition, might not only interact but also be the phenomenological expression of common brain networks. Strikingly, in the study Principal Component Analysis indicated that higher stress-sensitivity contributes positively to cognitive performance in the selected spatial learning task - a holeboard, that can be surprising if contrasted with "static" models of stressemotion-cognition interactions, which would predict high stress-responsiveness to be associated to impaired performance in associative tasks. However, this is precisely one of the crucial contributions of the study by Brinks et al. (2007), to show that high stressreactive individuals $(\mathrm{BALB} / \mathrm{c})$ can have an advantage over low stress-reactive individuals $(\mathrm{C} 57 \mathrm{BL} / 6 \mathrm{~J})$ to learn about certain relational tasks. As $\mathrm{BALB} / \mathrm{c}$ mice in the study were superior learners in the modified holeboard while they were previously shown to underperform C57BL/6J mice in the more stressful spatial learning water maze task, a linear relationship cannot explain the association between stress reactivity traits and perform- ance in relational tasks. Instead, Brinks et al. (2007) work - together with earlier reports supports the existence of an inverted $U$-shape to account for spatial learning under stress (Sandi and Pinelo-Nava, 2007). Presumably, the stress added by water immersion in the water maze task would render the "more stressed" BALB/c mice less cognitively effective to solve the stressful spatial challenge. Under such conditions, low stress-responsive individuals (such as C57BL/6J mice) would be benefited by the stress added by the physical characteristics of the task (Sandi et al., 1997). The emerging view underscores the individual stress response (and glucocorticoids as a plausible index; de Kloet et al., 1999; Roozendaal et al., 2002) as a critical factor (as opposed to the stressful context) to relate to learning performance.

The importance of taking individual differences into account to understand the neurobiological systems that mediate particular behaviors is becoming increasingly evident, even if such an approach is still far from being systematically incorporated. Brinks et al. (2007) study provides a clear example of its relevance and shows the benefit of using multivariate analysis approaches to understand complexity related to the neurobiology of behavior. The immediate challenge would be to formulate working models that permit organizing and manipulating such rich complexity in an effective and instructive manner for understanding how stress, emotion, and cognition intimately interact.

\section{REFERENCES}

Brinks, V., van der Mark, M., de Kloet, R., and Oitzl, M. (2007). Emotion and cognition in high and low stress sensitive mouse strains: a combined neuroendocrine and behavioral study in BALB/c and C57BL/6J mice. Front. Behav. Neurosci. 1, 8. doi: 10.3389/ neuro.08/008.2007.

de Kloet, E. R., Oitzl, M. S., and Joëls, M. (1999). Stress and cognition: are corticosteroids good or bad guys? Trends Neurosci. 22, 422-426.

Pessoa, L. (2008). On the relationship between emotion and cognition. Nat. Rev. Neurosci. 9, 148-158.

Phelps, E. A., and LeDoux, J. E. (2005). Contributions of the amygdala to emotion processing: from animal models to human behavior. Neuron 48, 175-187.

Richter-Levin, G., and Akirav, I. (2003). Emotional tagging of memory formation - in the search for neural mechanisms. Brain Res. Rev. 43, 247-256.

Roozendaal, B., Quiriarte, G. L., and McGaugh, J. L. (2002). Glucocorticoids interact with the basolateral amygdala beta-adrenoceptor - cAMP/cAMP/PKA system in influencing memory consolidation. Eur. J. Neurosci. 15, 553-560.

Sandi, C., Loscertales, M., and Guaza, C. (1997). Experience-dependent facilitating effect of corticosterone on spatial memory formation in the water maze. Eur. J. Neurosci. 9, 637-642.

Sandi, C., and Pinelo-Nava, M. T. (2007). Stress and memory: behavioral effects and neurobiological mechanisms. Neural. Plast. PMID: 18060012.

Received: 08 October 2008; published: 15 December 2008

Citation: Front. Neurosci. (2008) 2, 2: 134-135. doi: 10.3389/neuro.01.042.2008

Copyright: (C) 2008 Sandi. This is an open-access publication subject to an exclusive license agreement between the authors and the Frontiers Research Foundation, which permits unrestricted use, distribution, and reproduction in any medium, provided the original authors and source are credited.

\title{
Perinatal choline supplementation: a smart lifelong solution to age-related dementia
}

\author{
Ranier Gutierrez ${ }^{1 *}$ and Sidney A. Simon ${ }^{2}$ \\ Department of Pharmacology, CINVESTAV del IPN, Mexico City, Mexico \\ 2 Department of Neurobiology, Duke University, NC, USA \\ *Correspondence: ranier@cinvestav.mx
}

\section{A commentary on}

Developmental periods of choline sensitivity provide an ontogenetic mechanism for regulating memory capacity and age-related dementia

by Warren H.Meck, Christina L. Williams, Jennifer M. Cermak and Jan Krzysztof Blusztajn
Choline is an essential nutrient that is found in several foods (e.g. eggs and liver), and is synthesized in the body. Choline is an important constituent of phosphatidylcholine, a type of phospholipid found in membranes. As such, choline plays an important role in membrane structure and in membrane-mediated cell signaling via phospholipases. As a precursor to acetylcholine, and a neurotransmitter involved in muscle control, attention, learning and memory, it is important in many cognitive processes. Choline also plays a number of roles in brain development. Maternal reserves of choline 